\title{
Prolonged exposure as early intervention in an emergency department context: lessons learned from a terminated RCT.
}

Maria Bragesjö ( $\square$ maria.bragesjo@ki.se )

https://orcid.org/0000-0003-2246-3842

Filip Arnberg

National centre for disaster psychiatry

Erik Andersson

Karolinska Institutet

\section{Research note}

Keywords: Prolonged Exposure, Prevention, Post-traumatic stress disorder (PTSD), Intrusive memory, early intervention, acute stress disorder (ASD), memory consolidation

Posted Date: July 16th, 2019

DOI: https://doi.org/10.21203/rs.2.11136/v1

License: (c) (i) This work is licensed under a Creative Commons Attribution 4.0 International License. Read Full License 


\section{Abstract}

Objective The main purpose of the trial was to test if a brief trauma focused cognitive-behavior therapy (prolonged exposure; $\mathrm{PE}$ ) provided within $72 \mathrm{~h}$ after a trauma could be effective in decreasing the incidence of post-traumatic stress disorder (PTSD), replicating and extending findings from an earlier trial. After a pilot study $(\mathrm{N}=10)$, which indicated feasible and deliverable study procedures and interventions, we subsequently launched an RCT with a target sample size of 352 participants randomized to either three sessions PE or non-directive support. Due to an unforeseen major reorganization at the hospital, the RCT was discontinued after 32 included participants. Results In this paper, we highlight obstacles and lessons learned from our feasibility work, relevant for preventive psychological interventions for PTSD in emergency settings. One important finding was the high degree of attrition: only $78 \%$ and $34 \%$ respectively came back for the two months and six-months assessments. There were also difficulties in reaching eligible patients immediately after the event. Based on our experiences, we envisage that alternative models of implementation might overcome these obstacles, for example, with remote delivery of both assessments and treatment, combined with multiple recruitment procedures. Lessons learned from this terminated RCT are discussed in depth.

\section{Background}

Psychologically traumatic events will affect about $70 \%$ of the population [1]. A clinically substantial proportion, an estimated 5.6\% in Sweden, may develop post-traumatic stress disorder (PTSD) [1], which includes symptoms of re-experiencing, avoidance, cognitive and mood changes, and hyperarousal [2].

PTSD is a detrimental condition as it is associated with increased risk of suicide, drug and alcohol dependence, and sick leave [3], and increased somatic problems including neurological, vascular, respiratory, gastrointestinal, and autoimmune diseases $[4,5]$.

Despite the high societal and individual burdens associated with PTSD, only a fraction of cases are detected by the health care system [6] and evidence-based trauma-focused treatments for PTSD are seldom available in the real world setting $[7,8]$. One way to decrease the prevalence of PTSD is to intervene before the disorder develops [9]. A recent pilot trial $(\mathrm{N}=137)$ from the US by Rothbaum et al. [10] indicated that a modified version of prolonged exposure (PE; the first-line treatment for PTSD [11]) may be effective in preventing the onset of PTSD symptoms in emergency trauma care patients. In the Rothbaum et al. [10] study, treatment was initiated within 72 hours after trauma followed by two weekly sessions. Results showed that PE was superior to the assessment only control group in reducing PTSD symptoms 12 weeks after the event [10] and indicated that the treatment mitigated genetic predisposition to PTSD [12].

In 2016, our research group set out to replicate and extend the findings by Rothbaum et al. [10], originally with the aim to assess the effectiveness of the modified PE protocol in a larger sample, using an active control group of non-directive support $[13,14]$, blinded assessors, and longer follow up. After a pilot trial of 10 consecutive trauma patients, we subsequently launched a large-scale RCT. Given the 
estimated effect sizes in the previous trial by Rothbaum et al. we calculated that 352 participants (176 in each group) was needed in order to detect a standardized effect size (Cohen's $d$ ) of 0.3 (10\% data attrition allowed, two tailed tests, $p=.05,80 \%$ power). However, several barriers arose during recruitment and the RCT was terminated prematurely. In this paper, we describe the methods we used and the combined results from a total of 32 participants. We will highlight different implementation issues of delivering PE for psychological trauma patients within a hospital context and provide suggestions on how to resolve these issues.

\section{Methods}

\section{Pilot and main RCT design}

The pilot study of 10 participants and the subsequent large-scale trial used an identical randomized controlled design and procedures. Study participants were hospital patients who had experienced a potentially traumatic event within the last 72 hours. Participants were allocated to either modified PE or a control condition. The sample reported throughout this paper is the pooled sample of 10 participants from the pilot study and the first 22 from the RCT.

\section{Participants}

Eligible participants were Swedish-speaking patients over 16 years of age attending the emergency department (ED) at Karolinska University hospital in Solna, Sweden, within 72 hours after experiencing a psychologically traumatic event according to the DSM-5 criterion A for PTSD (i.e., exposed to: death, threatened death, actual or threatened serious injury, or actual or threatened sexual violence). Exclusion criteria are shown in the supplement. Common traumatic events that lead to attendance at the emergency department were motor vehicle accident and assaults.

\section{Procedure}

The recruitment included several steps. A pre-selection screening was done each weekday morning at an out-patient clinic nearby the ED by a clinical psychologist who scanned the digital medical records of newly arrived patients in order to assess criterion A and potential exclusion criteria. Potentially eligible patients were then assessed by one of four clinical psychologists involved in the study team either in the emergency room or at bedside in the hospital ward. Already discharged patients were interviewed via telephone and, if eligible, invited to the hospital to receive the intervention in the office of the clinical psychologist. Eligible patients received both written and verbal information about the study. After signing informed consent, the psychologist conducted the baseline assessments and subsequently opened the sealed envelope containing the random assignment to either PE or active control. The intervention was delivered immediately after randomization. Participants in both groups were assessed at week 1-3 (during 
treatment) and after 2 and 6 months. Primary endpoint was 6 months. The participants were not reimbursed for their participation in the study.

\section{Measures}

The primary outcome was PTSD symptom severity assessed using the Clinician Administered PTSD Scale for DSM-5 (CAPS-5) $[15,16]$. The assessors $(n=4)$ were clinical psychologists experienced in conducting structured diagnostic interviews. Each assessor had received extensive training, up to $2 \frac{1}{2}$ days, in administration and scoring of the CAPS-5. Inter-rater agreement was excellent (Inter Class Correlation between 0.91-0.98). Supervision was provided on difficult cases. Secondary outcomes were the PTSD Symptom Checklist for DSM-5 (PCL-5) [17], an intrusion diary [18, 19], Montgomery Åsberg Depression Rating Scale-Self rated version (MADRS-S) [20], the World Health Organization Disability Assessment Schedule-12 (WHODAS) [21], EQ-5D (Euroqol-5D) [22] and the Insomnia Severity Index (ISI) [23]. Detailed information about the outcome measures are shown in the supplement.

Interventions

Participants in both conditions received a three-session intervention in which the first session was provided immediately after the screening, which took place $<72$ hours after the traumatic event. The therapists in the RCT were five clinical psychologists with extensive experience and training in PE. All therapists received additional training (1 day) in the adapted PE protocol used in this study and also on how to deliver the control condition treatment. Supervision was provided when needed.

\section{Prolonged exposure (PE)}

The treatment protocol from the Rothbaum et al. [10] study was provided to us from the study authors. The protocol was translated to Swedish by the first author, who has been trained and certified in PE by the original treatment developer (Professor Edna Foa) and who prior to the study had worked clinically with PE for 17 years. A description of the treatment protocol is provided in the supplement.

\section{Control group}

The main purpose of the control group was to provide a credible intervention that is in line with international recommendations on early interventions after trauma $[13,14]$ : psychoeducation about common reactions after trauma and general non-directive support aimed at promoting safety, calmness, 
connectedness, self-efficacy and hope. The sessions followed the same structure and were matched to the intervention in frequency and duration.

\section{Randomisation and masking}

Block randomization was conducted by an independent party (Karolinska Trial Alliance) on a 1:1 ratio. The participants were randomized after the baseline assessment using sealed envelopes. The sealed envelopes were otherwise kept safe and accessible only to persons authorized to unblind. The randomization key was kept at the clinic. Participants were blinded to the conditions in order to control for expectation confounders. Therefore, participants were simply told that they would receive one of two treatments aiming to prevent the onset of PTSD.

\section{Results}

Study progression

Baseline characteristics for the included participants are presented in Table 1. Detailed information is provided in the supplement together with the study flow chart. Unforeseen major organizational changes at the hospital negatively affected the recruitment. At the start of the RCT (May 2018), $30 \%$ of the trauma patients at the ED were recruited into the current study whereas this figure decreased rapidly to only $2.8 \%$ after the organizational changes in the subsequent months (Figure S2, see supplement). All the trauma care moved to an entirely new hospital, new Karolinska University Hospital, designed for highly qualified specialized trauma care. The hospital was mandated to take on only very severely injured patients, leading to a markedly lower number of patients overall and a lower number of eligible patients for the study. Treatment adherence and credibility were rated as overall high but many participants dropped out at the follow-up assessments (see supplement).

Preliminary outcome data

SUD ratings for all three treatment sessions are shown in figure 1 and CAPS-5, PCL-5, and MADRS-S scores are shown in Table 2. Data from the intrusion diary are shown in the online supplement. No adverse events were reported by the participants that could be attributed to either of the interventions.

\section{Discussion}

The current study was initially designed as a as a preventive intervention for PTSD to replicate and extend of the Rothbaum et al. [10] trial. After a pilot trial, we aimed to recruit 352 patients at the ED in an 
RCT to test the effects of modified PE compared with an active control condition. The RCT started out with a fair recruitment rate of $30 \%$ of attending patients but was discontinued when the hospital underwent unforeseen major organizational changes and started to treat only severely injured patients, leading to a drastic decline in the eligible number of patients that could be recruited.

In this study, we took advantage of already established procedures and infrastructure at the hospital. For example, we used regular hospital psychologists and the hospital's medical record system at the ED department to find and approach eligible trauma patients. We considered this regular clinic approach a major strength in the study as it could enhance implementation after study completion. The reliance on personnel and infrastructure at one specific hospital unfortunately became a major obstacle in this study as recruitment rates fell significantly during the re-organization. In hindsight, we could have circumvented the problem of recruiting patients if we had developed an infrastructure and study procedures outside regular hospital routines that were able to function independently of organizational changes within the hospital.

A significant proportion of ED patients (24\%) in the current study were excluded due to the time criterion: Many patients had already returned home after the visit to the ED when we detected them in the medical record system and could thus only be scheduled for treatment the day after the traumatic event. Memory consolidation theory proposes that the optimal time to intervene on memory consolidation is within 6 hours after the traumatic event has taken place [24]. From that perspective, it is important to develop simple interventions that can be delivered to the patient at an early stage in the hospital as recently shown by lyadurai et al. [18]. Another option for future research would therefore be to recruit, assess, and treat trauma patients remotely, for example, by using mobile- or internet-delivered approaches to ensure swift and easy recruitment at the same time facilitating stability in study procedures independent of the current hospital organization. Internet-delivered psychological treatments have in general been shown to increase accessibility and can reach a wider population by distance, also when implemented in regular care [25]. An interesting future innovation would therefore be to investigate if it is possible to reach trauma patients remotely in the early aftermath of traumatic events. However, one large scale trial from 2013 showed relatively low compliance rates for an automated internet-based psychological preventive intervention for PTSD [26]. Many individuals recruited in this trial were not PTSD cases and may therefore not have been motivated to do any more follow-up assessments, as it might have been inconvenient (e.g., traveling to hospital, navigating to a new clinician, missing work). Remotely delivered easily accessible technological tools could potentially circumvent some of the high attrition data found in this study.

To summarize, this prematurely terminated trial generated important scientific and clinical experiences. Based on these experiences, we suggest that future research into the prevention of PTSD might benefit from considering implementation models with remotely-delivered, easily accessible interventions that are independent of regular health organization routines or more simple and easy deliverable interventions provided by non-specialists at the ED. 
Limitations

A major limitation with the study is the small sample size and limited follow up data. It still holds important feasibility aspects of the difficulty in reaching trauma victims early.

\section{Abbreviations}

CAPS-5: Clinician Administered PTSD Scale for DSM-5

ED: Emergency department

EQ-5D: (Euroqol-5D)

ISI: Insomnia Severity Index

MADRS-S: Montgomery Åsberg Depression Rating Scale-Self rated version

PCL-5: PTSD Symptom Checklist for DSM-5

PE: Prolonged Exposure

PTSD: Post-traumatic stress disorder

RCT: Randomized controlled trial

WHODAS: World Health Organization Disability Assessment Schedule

\section{Declarations}

Ethics approval and consent to participate

The study was registered at Clinicaltrials.gov (ID: NCT03116165) and approved by the Regional Ethical Review Board in Stockholm, Sweden (ID: 2015/1820-31). The study is reported in accordance to the CONSORT statement for nonpharmacological treatments.

Eligible patients received both written and verbal information about the study, and was not included in the study until after signing informed consent.

Consent for publication

Not applicable. 
Availability of data and material

The study protocol and data procedures used in the current study are available from the corresponding author on reasonable request.

Competing interests

F.K.A receives book royalties from Natur och Kultur for the Swedish translation of the prolonged exposure treatment manual. E.A and M.B report no competing interests.

\section{Funding}

The study was funded through The Swedish Research Council (grant 2016-02359), Swedish Society For Medicine (grant 658811) and Stockholm County Healthcare (grant 20170018). Neither of the funding organizations had any role in the conception of the study design or in the collection, analysis or interpretation of the data, in the writing of the report, or in the decision to submit the paper.

Authors' contributions

All authors contributed to the study design. M.B conducted the data collection under supervision from E.A. Data analysis and interpretation were done by M.B and E.A and they also did the first draft of the article. F.K.A thereafter contributed to writing the article. All authors made read and approved the final manuscript.

Acknowledgements

The authors express their sincere gratitude to Emily Holmes who provided a significant intellectual contribution to the design of this study.

\section{References}

1. Koenen, K.C., et al., Posttraumatic stress disorder in the World Mental Health Surveys. Psychol Med, 2017: p. 1-15.

2. Kupfer, D.J. and D.A. Regier, Neuroscience, clinical evidence, and the future of psychiatric classification in DSM-5. Am J Psychiatry, 2011. 168(7): p. 672-4. 
3. Kessler, R.C., et al., Posttraumatic stress disorder in the National Comorbidity Survey. Arch Gen Psychiatry, 1995. 52(12): p. 1048-60.

4. McFarlane, A.C., et al., Physical symptoms in post-traumatic stress disorder. J Psychosom Res, 1994. 38(7): p. 715-26.

5. Song, H., et al., Association of Stress-Related Disorders With Subsequent Autoimmune Disease. Jama, 2018. 319(23): p. 2388-2400.

6. Al-Saffar, S., P. Borga, and T. Hallstrom, Long-term consequences of unrecognised PTSD in general outpatient psychiatry. Soc Psychiatry Psychiatr Epidemiol, 2002. 37(12): p. 580-5.

7. Jonas, D.E., et al., AHRQ Comparative Effectiveness Reviews, in Psychological and Pharmacological Treatments for Adults With Posttraumatic Stress Disorder (PTSD). 2013, Agency for Healthcare Research and Quality (US): Rockville (MD).

8. Deacon BJ and F. NR, Therapist barriers to the dissemination of exposure therapy., in Handbook of treating variants and complications in anxiety disorders, M.D. Storch EA, Editor. 2013, Springer. p. 363-373.

9. Association, A.P., Diagnostic and statistical manual of mental disorders (DSM-5®). 2013: American Psychiatric Pub.

10. Rothbaum, B.O., et al., Early intervention may prevent the development of posttraumatic stress disorder: a randomized pilot civilian study with modified prolonged exposure. Biol Psychiatry, 2012. 72(11): p. 957-63.

11. Powers, M.B., et al., A meta-analytic review of prolonged exposure for posttraumatic stress disorder. Clin Psychol Rev, 2010. 30(6): p. 635-41.

12. Rothbaum, B.O., et al., Early intervention following trauma may mitigate genetic risk for PTSD in civilians: a pilot prospective emergency department study. J Clin Psychiatry, 2014. 75(12): p. 1380-7.

13. Juen, B., et al., The comprehensive guideline on mental health and psychosocial support (MHPSS) in disaster settings. 2016, OPSIC: Operationalising Psychosocial Support in Crisis.

14. World Health Organization, Guidelines for the management of conditions specifically related to stress. 2013, Geneva: WHO.

15. Foa, E.B., et al., The validation of a self-report measure of posttraumatic stress disorder: The Posttraumatic Diagnostic Scale. Psychological Assessment, 1997. 9(4): p. 445-451.

16. Weathers, F.W., et al., The Clinician-Administered PTSD Scale for DSM-5 (CAPS-5). 2013: National Center for PTSD

17. Blevins, C.A., et al., The Posttraumatic Stress Disorder Checklist for DSM-5 (PCL-5): Development and Initial Psychometric Evaluation. J Trauma Stress, 2015. 28(6): p. 489-98.

18. Iyadurai, L., et al., Preventing intrusive memories after trauma via a brief intervention involving Tetris computer game play in the emergency department: a proof-of-concept randomized controlled trial. Mol Psychiatry, 2018. 23(3): p. 674-682. 
19. Horsch, A., et al., Reducing intrusive traumatic memories after emergency caesarean section: A proofof-principle randomized controlled study. Behav Res Ther, 2017. 94: p. 36-47.

20. Svanborg, P. and M. Asberg, A new self-rating scale for depression and anxiety states based on the Comprehensive Psychopathological Rating Scale. Acta Psychiatr Scand, 1994. 89(1): p. 21-8.

21. Üstün, T.B., et al., Measuring health and disability: Manual for WHO disability assessment schedule WHODAS 2.0. 2010: World Health Organization.

22. Rabin, R. and F.d. Charro, EQ-SD: a measure of health status from the EuroQol Group. Annals of Medicine, 2001. 33(5): p. 337-343.

23. Bastien, C.H., A. Vallieres, and C.M. Morin, Validation of the Insomnia Severity Index as an outcome measure for insomnia research. Sleep Med, 2001. 2(4): p. 297-307.

24. McGaugh, J.L., Memory-a century of consolidation. Science, 2000. 287(5451): p. 248-51.

25. Titov, N., et al., ICBT in routine care: A descriptive analysis of successful clinics in five countries. Internet Interventions, 2018. 13: p. 108-115.

26. Mouthaan, J., et al., Internet-Based Early Intervention to Prevent Posttraumatic Stress Disorder in Injury Patients: Randomized Controlled Trial. Journal of Medical Internet Research, 2013. 15(8): p. e165.

\section{Tables}

Due to technical limitations, the tables have been placed in the Supplementary Files section.

\section{Figures}



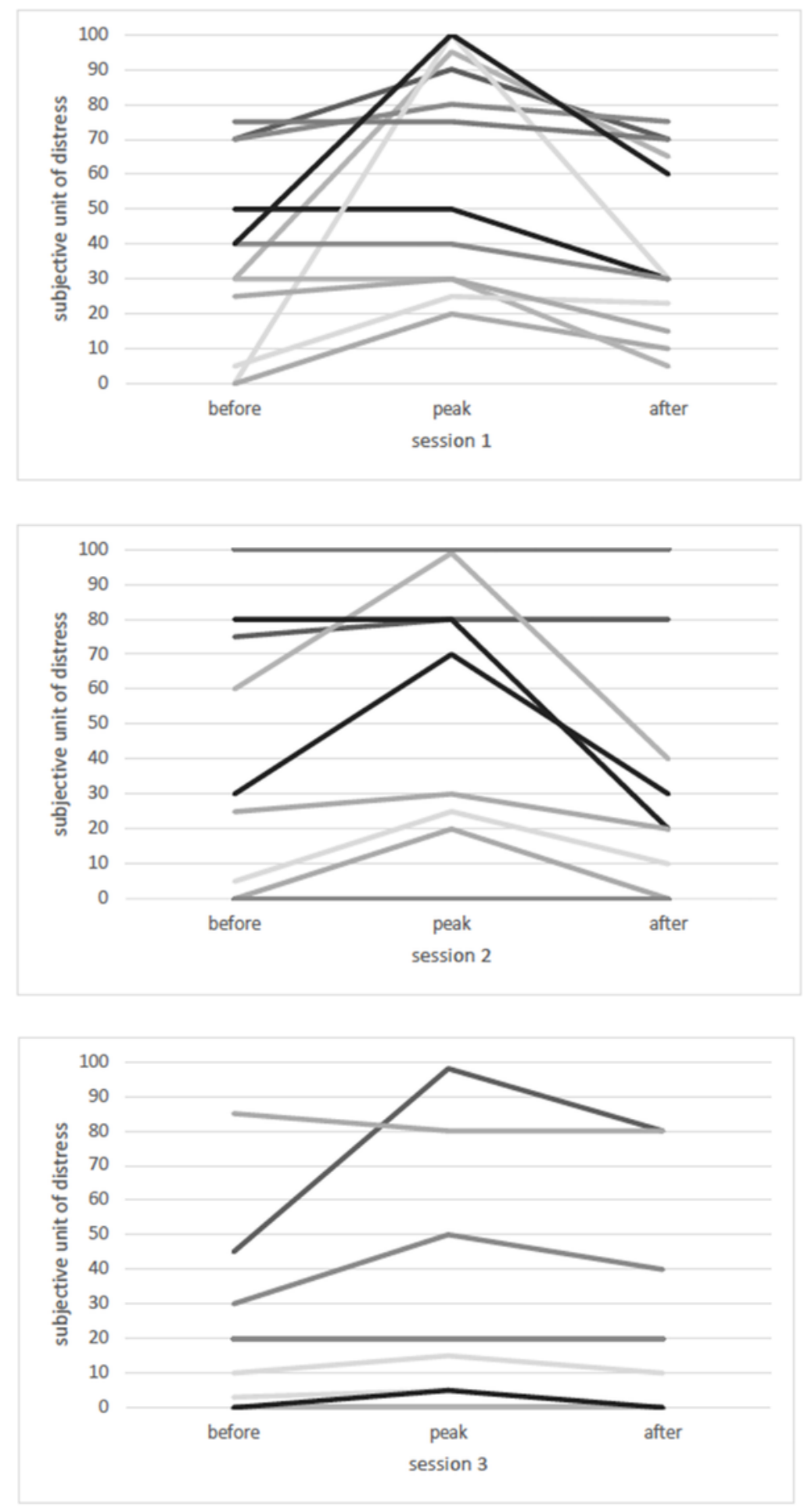

\section{Figure 1}

Individual subjective units of distress ratings across the treatment sessions. A decrease in the participants' subjective distress during treatment can be seen using mean SUD ratings (session 1 pre-SUD 35, peak-SUD 61, post-SUD 40; session 2 pre-SUD 42, peak-SUD 56, post-SUD 33; session three pre-SUD 24, peak-SUD 33, post-SUD 28). 


\section{Supplementary Files}

This is a list of supplementary files associated with this preprint. Click to download.

- SupplementLessonlearnedBragesjoArnbergAndersson.docx

- table2.pdf

- Table1.pdf

- CONSORTchecklistLessonslearnedBMCResearchnotes.doc 